\title{
Experience of Childbirth With Birth Ball: A Randomized Controlled Trial
}

\author{
Morvarid Ghasab Shirazi $^{1}{ }^{\circledR}$, Shahnaz Kohan ${ }^{2}$, Firoozeh Firoozehchian $^{1}$, Elham Ebrahimi $^{3^{*}(\mathbb{D}}$
}

\begin{abstract}
Objectives: Childbearing is considered as a great event in the lives of many women while the effect of pain on this event is undeniable. Thus, the think of pain and how to overcome it has engaged the minds of women, their family, and health-care providers. The birth ball is one of the non-invasive methods of pain control. Therefore, the present study aimed to evaluate the effect of the birth ball on the pain and self-efficacy of pregnant women during the childbirth process.

Materials and Methods: This study was a randomized clinical trial. A total of 178 participants were selected based on the specific selection criteria and randomly allocated to control and intervention groups. The women in the intervention group were asked to join a planned exercise with the birth ball including a 20-minute well-defined exercise three times a week for 6-8 weeks at home whereas those in the control group followed up the routine prenatal cares. The questionnaires were completed by the participants at the four and eight-centimeter cervical dilations.

Results: Based on the results, birth ball exercises could significantly improve childbirth self-efficacy and pain so that labour pain was lower in this group of women as compared to the other group $(P<0.001$ in both cervical dilatations). In addition, the score of selfefficacy was higher in the intervention group compared to the control group $(P<0.001)$. Further, the result of generalized estimating equation model showed that birth ball exercise can decrease the childbirth pain. However, part of this effect may be related to an increase in the patients' self-efficacy (30\%-40\%).

Conclusions: In general, although birth ball exercise could decrease the childbirth pain, part of this effect was probably associated with an increase in self-efficacy of the patients.

Keywords: Labour pain, Birth ball, Self-efficacy
\end{abstract}

\section{Introduction}

Although the effect of childbearing is evident, it is regarded as a prominent experience in the lives of various women. A lot of women describe that they were happier after delivery if they experienced less pain (1). Thus, thinking of the pain and its overcoming are among the challenges of women and their families, as well as their health-care providers (2). Today, there is a new perception toward managing the childbearing with physiological strategy worldwide, which is called physiological delivery. It is defined as a type of vaginal delivery that is safer for pregnant women who are in the labour wards with no medical intervention while in an anxiety-free atmosphere in which the mother's hormonal system augments the delivery (3). Therefore, there is a tendency toward using non-pharmacological pain-relieving methods for alleviating the pain of delivery.

A coping strategy is one of the strategies for pain control. In this method, the self-efficacy as a method of coping can empower the women to tolerate the pain and experience pleasant childbirth (4). Lowe believed this effect is probably related to the body and feeling improvement of the patients (5).

In addition, the birth ball is considered as another non-invasive method utilized for controlling the pain. The Swiss ball, known as the birth ball in the normal vaginal delivery wards, was first introduced in 1963 for neurodevelopment treatment purposes (6). It did not take long to use it as an instrument of childbirth in the 1980s. Perez and Simkin were the first ones to spread the knowledge of its use among the midwives and nurses or other health-care providers as a childbearing facilitator (7). They further explained its advantages as an applicable instrument for positioning and pain relieving during the childbirth process (8). Then, the use of the birth ball expanded and its related sports during childbirth were improved accordingly (9). The first scientists justified that the birth ball improves the mother's control over her body and builds body confidence. Furthermore, its role as a play can be like childhood memories $(8,9)$. Although the birth ball, as a simple and useful tool in maternity practice, was introduced many years ago, the published documents about its effects on childbirth outcomes are sparse. Accordingly, the current study sought to investigate the effect of the birth ball on the pain and self-efficacy of pregnant women during the process of childbirth.

\section{Received 8 February 2018, Accepted 11 June 2018, Available online 23 June 2018}

${ }^{1}$ Department of Midwifery, Guilan University of Medical Sciences, Rasht, Iran. ${ }^{2}$ Midwifery and Reproductive Health Department, Nursing and Midwifery Care Research Center, Faculty of Nursing and Midwifery, Isfahan University of Medical Sciences, Isfahan, Iran. ${ }^{3}$ Department of Reproductive Health, School of Nursing and Midwifery, Tehran University of Medical Sciences, Tehran, Iran. 


\section{Materials and Methods \\ Design}

This randomized clinical trial study was initiated according to a well-defined protocol. The participants were selected based on the specific selection criteria and randomly assigned to control and intervention groups. Moreover, the randomization process was based on a computer-generated block randomization list and was conducted in the shape of sequentially numbered in sealed envelopes which were generated by two study-free midwives. This survey lasted for one year and included 178 participants including 89 women in each group. The inclusion criteria included having a tendency for participation, being in 30-32 weeks of gestation, having a normal pregnancy, and having no history of a disease or obstetric complications based on a standard prenatal chart. Additionally, the exclusion criteria were not meeting the mentioned inclusion criteria, being forced to receive extra medical intervention such as epidural analgesia or emergency termination of pregnancy, or undergoing a cesarean section. Eventually, the online version of $\mathrm{G}^{*}$ Power software (Germany, version, 3.1.1) was employed to estimate the sample size (The study power $=0.8$, at the 0.05 significant levels).

\section{Instruments}

Three types of standard questionnaires were applied based on the purpose of the study. A questionnaire that involved demographic and obstetric information such as maternal age, educational status, job category, parity, and the like, visual analogue scale (VAS) as a rapid measurement of labour pain (10), and the childbirth selfefficacy inventory (CBSEI). The VAS is a ruler-shaped measure with a 10-centimeter horizontal line in which the numbers equal to the severity of pain. In addition, CBSEI (2) is a self-reporting scale that contains 26 items in two parallel subscales. The first 15 items are completed during the first stage of labour and the remaining 16 items are usually postponed to the second stage of labour (4). All questionnaires were completed between labour contractions.

\section{Study Intervention}

The protocol of the study was approved by Isfahan University of Medical Sciences under the number of IR.MUI.REC.1394.2.147. Moreover, this study was submitted to Iranian Registry of the Clinical Trials with the identical code of 20,147 and the ethical number of 1394.45.23.

After giving the study participants and hospital midwives, the study was initiated at Hajar hospital in Shahrekord. After obtaining written constant forms, the mothers in the intervention group were requested to attend the training class. Then, the balls were provided in the size of 55,65 , and $75 \mathrm{~cm}$ and mothers were guided to select the proper ball size according to their body size.
Next, 4 types of position with eight exercises were taught including sitting (pelvic rocking-forward and back, Hula-Hula-side-to-side, and rocking), standing (leaning forward on the ball and leaning against the ball on the wall-up and down), kneeling (hugging the ball and pelvic rocking), and squatting (leaning against the ball on the wall). All participants in the intervention group were advised to practice at home (at least twenty minutes three times a week for a period of 6-8 weeks).

\section{Data Collection and Analysis}

When the participants arrived at the hospital for childbirth, the nurses in labour units informed the research investigators. The nursing and midwifery, as well as the routine antenatal care was provided by hospital staff nurses while the research investigators only collected the required data. Women in the experimental group were given a birth ball to use during the labour and encouraged every hour to choose the most comfortable positions, movements, and exercises. Eventually, all the participants answered CBSEI and the short form of the McGill pain (SF-MPQ) questionnaires at two specific times (i.e., when cervical dilation reached 4 and 7-8 centimeters). The standard cares were provided for both groups

\section{Results}

As shown in Figure 1, a total of 312 participants initially entered the study.

Based on the result of Table 1, there are no significant differences between the study participants regarding their baseline characteristics.

Pain intensity was one of the important variables in this study. The results of Table 2 show that labour pain is lower in the birth ball exercise group compared to the control group $(P<0.001$ in both cervical dilatations). In addition, the score of self-efficacy of intervention group is higher than that of the control group $(P<0.001)$.

After controlling the factors that may alter the pain intensity with generalized estimation equations, the means of VAS scores $(\beta=1.85)$ were found to be significantly lower in the intervention group $(P<0.001)$ compared to the control group (Table 3 ). Women with higher selfefficacy scores had lower pain scores $(P<0.005)$.

Furthermore, the result of the Sobel test (Table 4) indicates that self-efficacy has a significant mediating effect on the relationship between birth ball exercises and childbirth pain. Sobel test further demonstrates that approximately $29.8 \%$ of the outcome variances is mediated by self-efficacy $(P<0.001)$.

\section{Discussion}

The results of the current study verified the positive role of exercise with the birth ball in decreasing the pain of childbirth. According to the literature, the fear of pain is the most important factor in preventing vaginal delivery. Melzack et al found that the ball can modify the pressure 


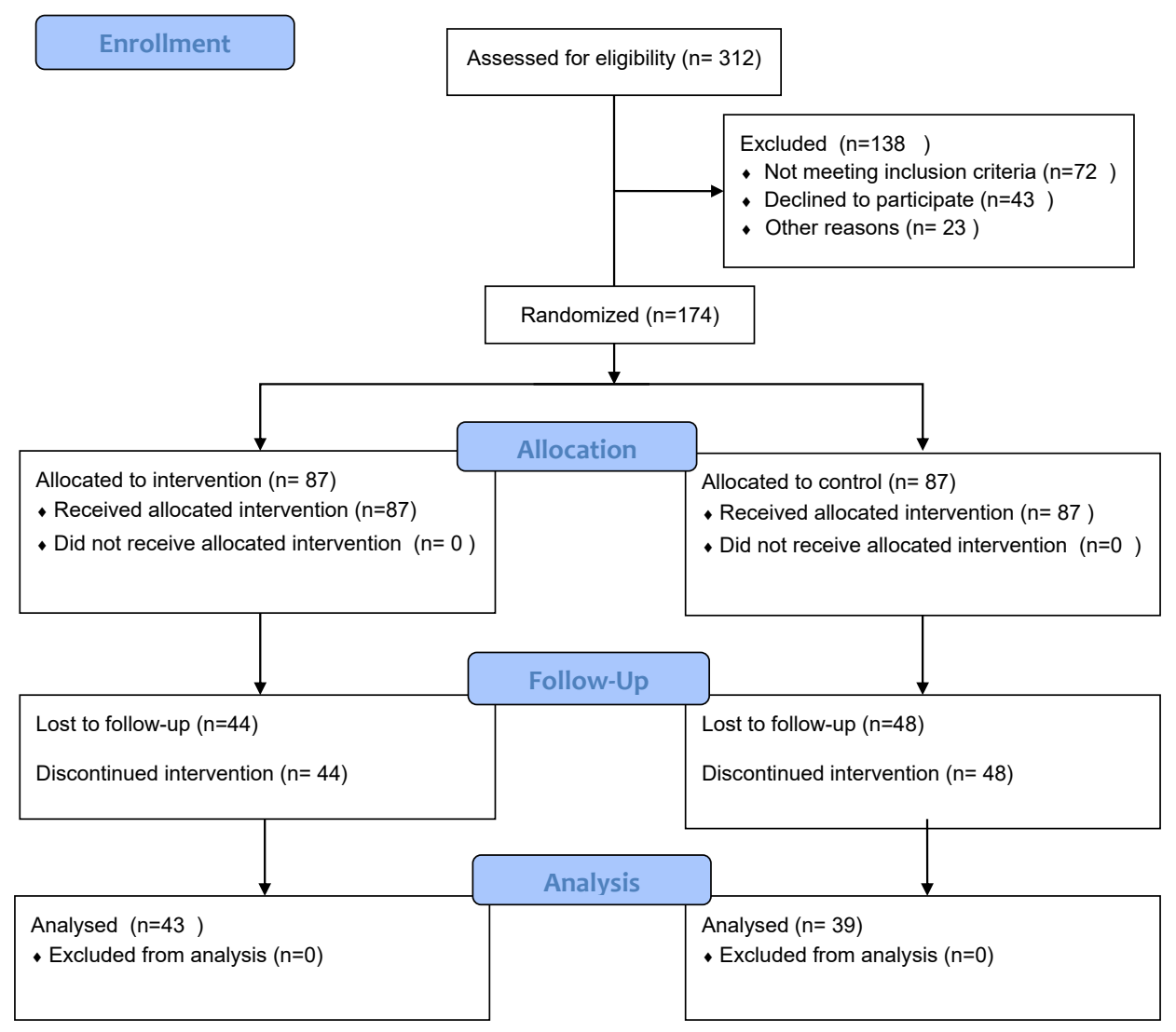

Figure 1. Flowchart of the study.

Table 1. The Characteristics of Study Groups

\begin{tabular}{|c|c|c|c|}
\hline Variable & Birth Ball Group ( $n=43$ ) & None Experimental Group ( $n=39)$ & $P$ value \\
\hline Age $(y)$, mean $\pm S D^{a}$ & $33.4 \pm 4.3$ & $32.23 \pm 3.9$ & 0.47 \\
\hline Duration of upright position (min), mean (SD) & $178.9 \pm 73.12$ & $104 \pm 78.95$ & 0.001 \\
\hline Duration of first stage labour (min), mean (SD) ${ }^{a}$ & $397 \pm 164.32$ & $423 \pm 245.54$ & 0.003 \\
\hline Duration of second stage labour (min), mean (SD) ${ }^{\mathrm{a}}$ & $29.17 \pm 21.3$ & $33.12 \pm 13.2$ & 0.653 \\
\hline Newborn baby weight (g), mean (SD), mean (SD) & $3021.371 \pm 432.19$ & $3042 \pm 412.34$ & 0.564 \\
\hline \multicolumn{4}{|l|}{ Education Level, No. (\%) } \\
\hline Illiterate & 4 & 3 & \multirow{4}{*}{3.46} \\
\hline Primary & 7 & 12 & \\
\hline Intermediate and high school, No. (\%) & 19 & 17 & \\
\hline University & 13 & 7 & \\
\hline \multicolumn{4}{|l|}{ Parity, No. (\%) } \\
\hline Primiparous & 28 & 21 & \multirow[t]{2}{*}{2.35} \\
\hline Multiparous & 15 & 18 & \\
\hline \multicolumn{4}{|l|}{ Induction, No. (\%) ${ }^{\mathrm{b}}$} \\
\hline Yes & 27 & 27 & \multirow[t]{2}{*}{0.007} \\
\hline No & 16 & 12 & \\
\hline \multicolumn{4}{|l|}{ Birth mode No. $(\%)^{c}$} \\
\hline Vaginal birth & 40 & 35 & \multirow[t]{2}{*}{0.768} \\
\hline Assisted vaginal birth & 3 & 4 & \\
\hline \multicolumn{4}{|l|}{ Apgar score (5 min) No. (\%)c } \\
\hline 9 & 40 & 32 & \multirow[t]{2}{*}{0.06} \\
\hline 10 & 3 & 8 & \\
\hline
\end{tabular}

${ }^{\mathrm{a}}$ Independent $t$ test; ${ }^{\mathrm{b}} \chi 2 ;{ }^{\mathrm{c}}$ Fisher exact test. 
Table 2. Pain and Self-efficacy Between the Groups

\begin{tabular}{lcccc}
\hline Variable & Overall Mean (SD) & Experimental Group Mean (SD) & Control Group Mean (SD) $^{\text {Statistic }^{\mathrm{a}}}$ & $\boldsymbol{P}_{\text {value }}$ \\
\hline Pain scales & & & & \\
VAS $(4 \mathrm{~cm})$ & $5.3(1.6)$ & $4.6(1.3)$ & $9.01(1.4)$ & -5.3 \\
VAS $(8 \mathrm{~cm})$ & $8.4(1.4)$ & $6.45(1.4)$ & & $0<0.001$ \\
Self-efficacy scales & & & & \\
Self-efficacy $(4 \mathrm{~cm})$ & $234(32.3)$ & $245(19.5)$ & $174(13.6)$ & -6.34 \\
Self-efficacy $(8 \mathrm{~cm})$ & $145.7(26.9)$ & $164.9(23.5)$ & $123.4(21.05)$ & -6.43 \\
\hline
\end{tabular}

VAS: Visual analogue scale.

${ }^{a}$ The Mann-Whitney $U$ test.

Table 3. Generalized Estimating Equations Model ${ }^{\mathrm{a}}$ on the Effect of Pain Score for Birth Ball Exercise Based on VAS

\begin{tabular}{lccc}
\hline Variables & $\boldsymbol{\beta}$ & SE & $\boldsymbol{P}$ value \\
\hline Group (experimental versus control) & -1.85 & 0.21 & $<0.001$ \\
\hline Time (cervical dilatation $8 \mathrm{~cm}$ versus $4 \mathrm{~cm})$ & 1.76 & 0.18 & $<0.001$ \\
\hline Self-efficacy & -0.03 & 0.003 & $<0.001$ \\
\hline
\end{tabular}

VAS: Visual analogue scale; SE, standard error.

a The model adjusted for methods of childbearing, oxytocin use, the length of the 1st labour stage, the time of upright position

on the perineum (11-13). In this regard, Simkin and Shilling related the birth ball effects to the freedom of movement that makes the child enter for birthing with an appropriate position (8). In line with the results of the present study, Leung et al concluded that the birth ball exercise can significantly decrease the labour back pain and pressure during the childbearing process $(P<0.001)$ (14).

Moreover, the results revealed that birth ball practice can increase self-efficacy during delivery. This effect may be due to the mechanisms that are responsible for coping skills which help to overcome the pain (9). Additionally, the birth ball may facilitate the sacroiliac and lumbosacral joints mobilization, as well as the oblique and transverse muscles tones thus it can help the pregnant mothers to manage their birth with good postures $(14,15)$. Similarly, McCrea and Wright suggested that birth ball exercise can alleviate labor pain by heightening the positive emotional feelings in women (16). In addition, Lowe showed lower degrees of childbearing pain by performing birth ball exercise and concluded that the self-efficacy can have a $30 \%-40 \%$ mediating effect on labour pain, which is consistent with the results of the current study (5).

The present research is one of the first studies that is conducted in this field although it has some limitations. Considering a sample size of only 312 women, controlling the confounding factors seems difficult. This effect remains after concerning the variables. Blinding is the other limitation of the study. There was no possibility of blindness in this study, which, in turn, could have had an impact on the results. Several researchers used the birth ball for pain relief and their participants performed the exercise for 6-8 weeks (17-20). However, the amount of $P$ value and the employed statistical methods differed between the present study and the above-mentioned studies. Further, the pain score in this study was lower compared to the other studies, $(P \leq 0.001$ vs. $P \leq 0.01)$, which may be related to the times of exercise (i.e., three times per week in the present study in comparison with two times per week in the other studies). Moreover, the current study used logistic regression that showed a pain decrease regarding the confounders such as upright position, cesarean, or vaginal delivery method, as well as oxytocin administration and the length of the 1st stage of childbearing. Finally, the present study evaluated the effect of the birth ball on the self-efficacy of women in the labour ward that is a wealthy variable for both patients and health-care providers.

\section{Conflict of Interests}

Authors declare that they have no conflict of interests.

\section{Ethical Issues}

The protocol of the study was approved by Isfahan University of Medical Sciences under the number of IR.MUI.REC.1394.2.147. Moreover, this study was submitted to Iranian Registry of the Clinical Trials with the identical code of 20,147 and the ethical number of 1394.45.23.

\section{Financial Support}

Isfahan University of Medical Sciences supported this study.

Table 4. The Mediated Effect of Self-efficacy on the Relationships Between Birth Ball Exercise and Childbirth Pain

\begin{tabular}{|c|c|c|c|c|c|c|}
\hline \multirow{2}{*}{ Dependent Variable } & \multirow{2}{*}{ Predictor } & \multirow{2}{*}{ Mediator } & \multicolumn{4}{|c|}{ Sobel Test } \\
\hline & & & Sobel Test Statistic & Indirect Effect & Ratio & Mediation (\%) \\
\hline VAS AND Self-efficacy & (Experimental vs. control groups) & Self-efficacy & $-3.23^{*}$ & -0.32 & 0.38 & 29.87 \\
\hline
\end{tabular}

VAS: Visual analogue scale.

a $P<0.001$. 


\section{Acknowledgments}

Therefore, we would like to thank Vice-chancellors of Education and Research and Technology Center of Isfahan University of Medical Sciences for financial support.

\section{References}

1. Waldenstrom U. Women's memory of childbirth at two months and one year after the birth. Birth. 2003;30(4):248254.

2. Gibbins J, Thomson AM. Women's expectations and experiences of childbirth. Midwifery. 2001;17(4):302-313. doi:10.1054/midw.2001.0263

3. Declercq ER, Sakala C, Corry MP, Applebaum S. Listening to mothers II: report of the second national US survey of women's childbearing experiences: conducted January-February 2006 for childbirth connection by Harris Interactive(R) in partnership with Lamaze International. J Perinat Educ. 2007;16(4):9-14. doi:10.1624/105812407x244769

4. Williams CE, Povey RC, White DG. Predicting women's intentions to use pain relief medication during childbirth using the Theory of Planned Behaviour and Self-Efficacy Theory. J Reprod Infant Psychol. 2008;26(3):168-179. doi:10.1080/02646830701691350

5. Lowe NK. Maternal confidence for labor: development of the Childbirth Self-Efficacy Inventory. Res Nurs Health. 1993;16(2):141-149.

6. Carriere B. The Swiss ball: theory, basic exercises and clinical application. New York: Springer; 1998.

7. Perez P. Birth balls: use of physical therapy balls in maternity care. East Johnson, VT: Cutting Edge Press; 2000.

8. Simkin P. Reducing pain and enhancing progress in labor: a guide to nonpharmacologic methods for maternity caregivers. Birth. 1995;22(3):161-171.

9. Watkins SS. Get on the ball-the 'birth ball' that is! Int J Childbirth Educ. 2001;16:17-19.

10. Chen SH. Effects of posture education program on women's pain perception and self-efficacy during labour. Kaohsiung: Kaohsiung Medical University; 1998.

11. Melzack R, Belanger E, Lacroix R. Labor pain: effect of maternal position on front and back pain. J Pain Symptom Manage. 1991;6(8):476-480. doi:10.1016/08853924(91)90003-M

12. Melzack R, Wall PD. Pain mechanisms: a new theory. Science. 1965;150(3699):971-979.

13. Shilling T, Difranco J, Simkin P. \#2: Freedom of Movement throughout Labor. J Perinat Educ. 2004;13(2):11-15. doi:10.1624/105812404x109483

14. Leung RW, Li JF, Leung MK, et al. Efficacy of birth ball exercises on labour pain management. Hong Kong Med J. 2013;19(5):393-399. doi:10.12809/hkmj133921

15. Ip WY. The effect of a theory-based intervention on promoting self-efficacy for childbirth among pregnant women in Hong Kong. The Chinese University of Hong Kong; 2005.

16. McCrea BH, Wright ME. Satisfaction in childbirth and perceptions of personal control in pain relief during labour. J Adv Nurs. 1999;29(4):877-884. doi:10.1046/j.13652648.1999.00961.x

17. Mirzakhani K, Hejazinia Z, Golmakani N, Sardar MA, Shakeri MT. The effect of birth ball exercises during pregnancy on mode of delivery in primiparous women. J Midwifery Reproductive Health. 2015;3(1):269-275. doi:10.22038/jmrh.2015.3562

18. Hau WL, Tsang SL, Kwan W, et al. The use of birth ball as a method of pain management in labour. Hong Kong J Gynaecol Obstet Midwifery. 2012;12(1):63-68.

19. Gau ML, Chang CY, Tian SH, Lin KC. Effects of birth ball exercise on pain and self-efficacy during childbirth: a randomised controlled trial in Taiwan. Midwifery. 2011;27(6):e293-e300. doi: 10.1016/j.midw.2011.02.004.

20. Makvandi S, Latifnejad Roudsari R,Sadeghi R, Karimi L. Effect of birth ball on labor pain relief: A systematic review and meta-analysis. J Obstet Gynaecol Res. 2015;41(11):1679-86. doi: 10.1111/jog.12802.

(c) 2019 The Author (s); This is an open-access article distributed under the terms of the Creative Commons Attribution License (http://creativecommons.org/licenses/by/4.0), which permits unrestricted use, distribution, and reproduction in any medium, provided the original work is properly cited. 